\title{
All-Digital Self-Interference Cancellation Technique for MIMO Full-Duplex Systems
}

\author{
J. Soma Sekhar \\ M. Tech, E.C.E Department \\ Gudlavalleru Engineering College \\ Gudlavalleru, A.P, India
}

\author{
E. V. Vijay \\ Assistant Professor, \\ E.C.E Department \\ Gudlavalleru Engineering College
}

\begin{abstract}
In this paper, the MIMO full-duplex system is proposed to remove self-interference signal using modified DFT based channel estimation technique. In SISO full-duplex systems only single transceiver can transmit data on either side simultaneously whereas in MIMO full-duplex systems multiple transceivers can transmit data simultaneously from both sides. During MIMO full-duplex transmission selfinterference and circuit impairments may occur, to avoid such noises digital cancellation technique is used which is a lowest complexity self-interference cancellation technique in all fullduplex systems. In this technique receiver auxiliary chain and its copy circuit called ordinary receiver chain is used to cancel out the both self-interference and its circuit impairments. Noise added during wireless channel is suppressed by using passive suppression technique and Gaussian noise, phase noise and quantization noise is eliminated by the active cancellation technique. So, combination of active cancellation and passive suppression techniques are used to eliminate the circuit impairments. The proposed channel estimation technique is used to cancel out the self-interference produced at the output of both auxiliary and ordinary receiver chains. By using digital self-interference cancellation technique the amount of self-interference cancelled is $37 \mathrm{dBm}$ higher than the receiver noise floor. The achievable rate and throughput of MIMO full-duplex system is $28 \mathrm{bits} / \mathrm{sec} / \mathrm{Hz}$ and 1049.04 Mbps respectively which is very large when compared to MIMO half-duplex , SISO full-duplex and SISO half-duplex systems.
\end{abstract}

\section{General Terms}

QAM, OFDM, Modified DFT based channel estimation, Digital cancellation.

\section{Keywords}

Full-duplex system, Digital self-interference, MIMO, Channel capacity, Throughput, Spectral efficiency

\section{INTRODUCTION}

The exponential evolution in mobile data traffic demands a spectrum and power efficient wireless transceiver which is capable to keep up this growth. To meet this demand, the solution was using high amount of spatial streams, efficient modulation schemes like 16 QAM, enhanced channel codes etc. But, there is one essential feature of wireless transceivers which was not seen anywhere is duplexity. Full-duplex wireless devices use the same frequency band for simultaneous transmission and reception. Recently, this concept has gained much attraction in the research community, since the capacity [10] of a wireless link might be doubled with respect to half-duplex systems.

The main constraint impacting full-duplex transmissions [5] is managing the strong self-interference signal which transmits and receives the signal simultaneously by using the same transceiver. For a full-duplex system to reach its greater spectral efficiency, the distorted signal is to be significantly blocked to the receiver reference noise floor. Self-interference cancellation technique is categorized into two types: passive suppression and active cancellation techniques. In passive suppression technique [4], the self-interfered signal is blocked before it passed through receiver circuitry. In case of active cancellation technique [7], the unwanted signals are removed by using digital cancellation technique [1], [2]. Before the signal entered into the receiver section nonlinearity is reduced by using the RF-power amplifier whose output signal is linear for most of the input signal and phase noise [3] is reduced by using PLL circuit.

The remaining of the paper is planned as follows: Design of MIMO full duplex system is discussed in section II. Section III deals with the experimental results and analysis of the proposed MIMO full duplex system. Section IV deals with the calculation of the system parameters called achievable rate, channel capacity, throughput and spectral capacity of the proposed MIMO full duplex system. Finally, Section V derives the conclusion.

\section{SYSTEM MODEL}

The complete system prototypical of proposed MIMO fullduplex system [6] architecture is displayed in fig 1. The system prototypical consists of both ordinary and auxiliary receiver chains which are used to cancel out the selfinterference signal. At the transmitter side, both information signals $\mathrm{X}_{1}$ and $\mathrm{X}_{2}$ are modulated and converted into time domain signals using OFDM. The time domain signal is then filtered using LPF, amplified using PA and power divided signals are transmitted through the transmitting antennas. The amount of divided signal is then given to both auxiliary and ordinary chains equally. The auxiliary and ordinary receiver chains are frequency converted and share the same PLL to reduce phase noises.

The output of both auxiliary and the ordinary receiver chains are given to proposed channel estimator to eliminate noise for large extent. Now, the estimated channel is multiplied with the auxiliary receiver output signal and then subtracted from the ordinary received signal to obtain interference-less signal. The output of the digital cancellation signal is QAM demodulated to get original signals $\mathrm{Y}_{1}$ and $\mathrm{Y}_{2}$. 


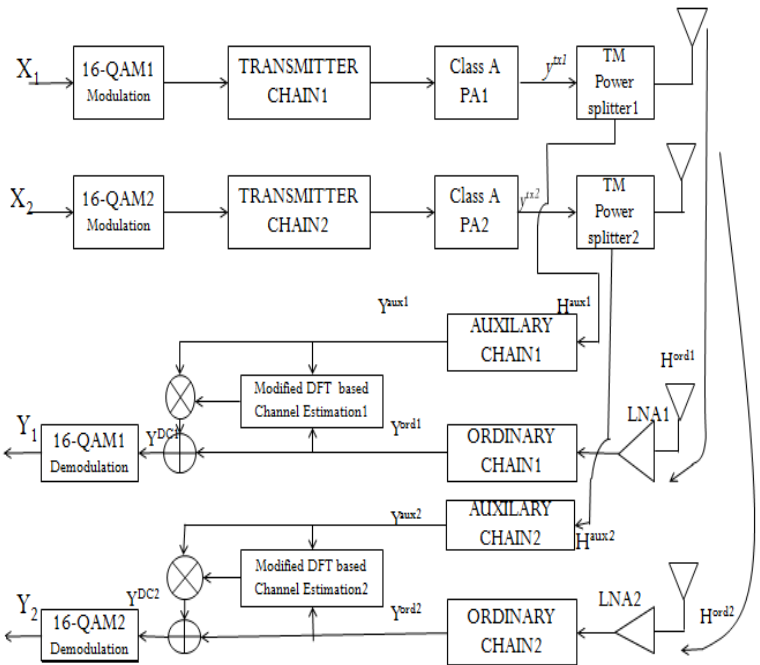

Fig 1: Complete block diagram of the proposed MIMO full duplex system

\subsection{QAM}

In QAM single carrier which is generated by a local oscillator circuit is divided equally into two paths, one path is delayed by 90 degrees called quadrature phase component and another path is a direct path called in phase component. Both are amplitude modulated and added together in a summing circuit to get information on both amplitude and phase of the transmitted carrier.

\subsection{Transmitter Chain}

The transmitter chain consists of OFDM, DAC, mixer along with PLL and filter. OFDM transmits huge amount of digital time domain non-overlapping narrow band subcarriers which are closely placed to each other's. Orthogonality between subcarriers is achieved by using IFFT at the transmitter side and FFT at the receiver side respectively [8]. IFFT converts digital frequency domain signals into digital time domain signals for base band wireless applications and FFT is used to convert digital time domain signals into digital frequency domain signals to make analysis easy. The one fourth length of IFFT is appended at the starting of the previous symbol called cyclic prefix or guard interval to lessen ISI between present and previous symbols during multipath propagation. DAC converts digital signal into an analog signal for base band wireless applications.

\subsection{Power Amplifier}

Power amplifier is an important module in modern wireless communication systems to increase the signal strength before it is processed through the wireless channel. The point at which output power is decreased at certain input level is called 1-dB Compression Point [12]. The power amplifier input and output relation diagram is displayed in below fig 2 .

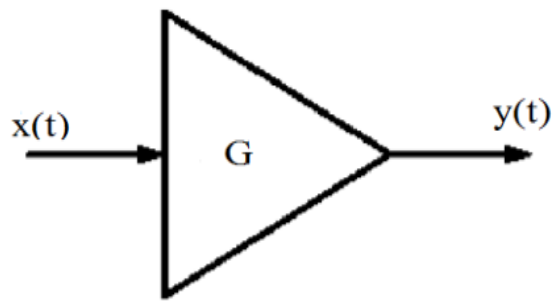

Fig 2: power amplifier input and output relation diagram
The system equation of linear power amplifier is taken as

$\boldsymbol{y}(\mathrm{t})=\beta_{1} \mathrm{x}(\mathrm{t})$

Due to the effect of non-linearity the overall equation of the RF power amplifiers is generalized as

$y(t)=\beta_{1} x(t)+\beta_{3} x(t)^{3}$

When a sinusoidal signal is applied to the input signal then the equation (2) can be taken as

$y(t)=\beta_{1} x(t)+\frac{3}{4} \beta_{3} x(t) A^{2}$

\subsection{Power Splitter}

Power splitter splits single input signal into the two equal signals. The direction of power flow is normal to the plane containing both $\mathrm{E}$ and $\mathrm{H}$ components.

$P_{z}=\frac{1}{2}\left|\vec{E}_{x} \times \vec{H}_{y}\right|$

In Transverse Magnetic (TM) case Magnetic vector $\left(\mathrm{H}_{\mathrm{x}}\right.$ or $\left.\mathrm{H}_{\mathrm{y}}\right)$ is normal to the direction of propagation while Electric vector has both normal and parallel components $\left(\mathrm{E}_{\mathrm{zx}}\right.$ or $\left.\mathrm{E}_{\mathrm{zy}}\right)$. So, under PML condition the reflection quotient $\left(R_{p}\right)$ is zero when it interface normal to either $\mathrm{x}$-axis or $\mathrm{y}$-axis. When a plane wave propagating along y-axis, PML medium absorbs both $H_{y}$ and $E_{z x}$ components [13] and allows $H_{x}$ and $E_{z y}$ components. When a plane wave propagating along $\mathrm{x}$-axis, PML medium absorbs both $H_{x}$ and $E_{z y}$ components and allows $H_{y}$ and $E_{z x}$ components.

\subsection{Wireless Channel}

Rayleigh fading [9] prototypical is mainly convenient in the circumstances where the signal is dispersed between the transmitter and receiver, which means signal spreads in multipath broadcasts. The real and imaginary parts of the response are demonstrated by

$r(t)=x(t)+j y(t)$

The signal is randomly attenuated and shifted in phase are expressed as

$R(t)=\sqrt{\left(x(t)^{2}+y(t)^{2}\right)}$

$R(\varphi)=L(x(t)+j y(t))$

\subsection{LNA}

LNA is used to strengthen the received RF signal from the wireless channel to an acceptable level and minimizing the noise added to it. The noise is minimized and signal strength is increased by using the stability analysis [11] and matched network selection. The 'BJT AT-31011' has better stability on the required frequency range of $2.4 \mathrm{GHz}$. By using two port network concept input reflection coefficient, output reflection coefficient and power available at the output of LNA have been calculated.

\subsection{Proposed Modified-DFT Based \\ Channel Estimation Technique}

The LS estimation is the modest channel estimation technique based on the parallel Gaussian channel model in frequency domain. Since the LS channel estimation technique [14] does not use any channel information its performance is not up to acceptable level. So, the proposed modified LS channel estimation technique called modified DFT-based channel 
estimation technique has been used because of lowcomputational complexity.

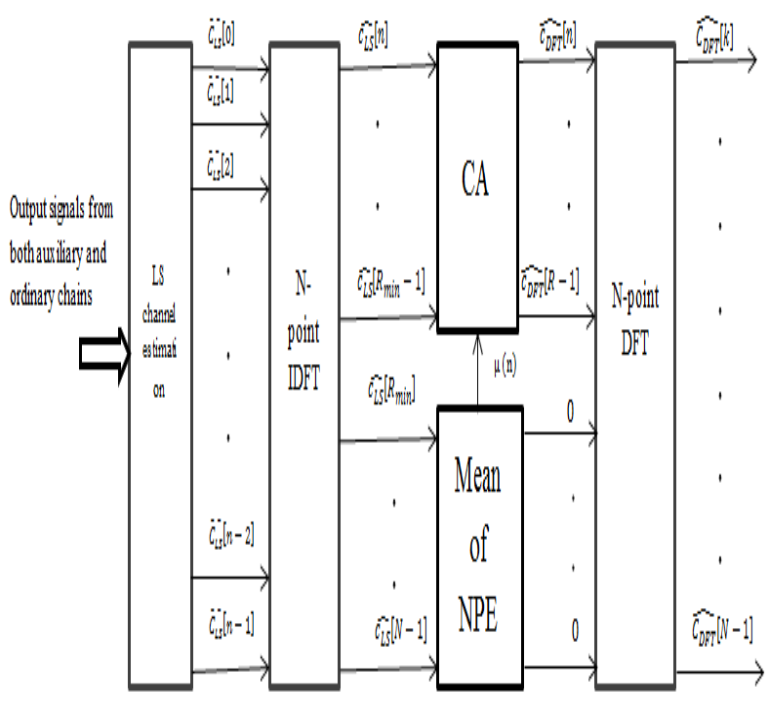

Fig 3: Block diagram of modified DFT-based channel estimation

The LS channel output signal has both original signal and noise signal. According to DFT-based channel estimation technique it takes the output values up to the length of the CIR $R$ whose length is less than the length of the guard interval and the remaining signal except length of the CIR is taken as zeros because it has noise only components. This technique also does not give the proper information but better than LS channel estimation. Now, the proposed modified DFT-based channel estimation technique displayed in above fig 3 consists of Channel Aggregator (CA) and mean of Noise Power Estimator (NPE) gives the proper channel information. The noise components which are directly taken zeros in the previous technique have some amount of information. So, that noise components are averaged and added to the CA to improve the signal information.

The mean of NPE first average all the time domain NPE noise-only existing part and added to the CA. The expression for NPE is given by

$\sigma_{\text {noise }}^{2}(n)=\frac{1}{N-R_{\min }} \sum_{n=R_{\min }}^{N-1}\left|\widehat{c_{L S}}[n]\right|^{2}$

Now, the mean of NPE is expressed as

$\mu(n)=\frac{1}{N-R_{\min }} \sum_{n=R_{\text {min }}}^{N-1}\left(\sigma_{\text {noise }}^{2}(n)\right)$

Where,

$$
\mu(\mathrm{n})=\text { mean of NPE }
$$

The discrete Mean Square Error (MSE) of modified DFTbased channel estimation technique of $n^{\text {th }}$ subcarrier is expressed as

$M S E_{\text {prop }}[n]=\frac{\left(R_{\min }+1\right)^{2}}{(K-2)^{3}} \frac{\gamma^{*}}{\left(\frac{S}{N}\right)}$

Where,

$$
R_{\text {min }}=\text { minimum value among the lengths of CIR }
$$

The MSE value is depends on the length of the CIR. If the length of the CIR is maximum $\left(\mathrm{R}_{\max }\right)$ then the maximum MSE is obtained. So, in the proposed modified-DFT based channel estimation technique minimum CIR ( $\left.\mathrm{R}_{\max }\right)$ length is taken to obtain the least MSE value.

\section{EXPERIMENTAL RESULTS and ANALYSIS}

The QAM output signal has a complex constellation points (I $+\mathrm{jQ}$ ) which are normally organized in a square set-up with equal vertical and horizontal spacing between them. Each point in a 16 QAM has separate amplitude and phase information (for example the point 0000 in a 16 QAM has amplitude value 0.9 volts and phase value -135 degrees and so on for all other 15 points). In OFDM, IFFT is used to convert digital frequency domain samples into digital time domain samples which is having 1000 symbols with each symbol of 64 bits ( 52 subcarriers +12 pilot carriers). So, 64 IFFT is used for this conversion process. Each symbol among 1000 symbols is separated by $1 \mathrm{~ms}$ and every symbol has 64 bits that each bit is separated by $15 \mathrm{~ms}$. So, overall each bit from total of 1000 symbols is separated by $15 \mu \mathrm{s}$. As each bit is separated by some seconds all the bits are non-overlap to each other. So, by using IFFT in OFDM all the bits are transmitted orthogonally. The end of the OFDM symbol is copied into the guard interval and this guard interval is transmitted followed by OFDM symbol. So, $25 \%$ at the end of a present symbol is copied and appended at the starting of that symbol itself to eliminate the inter symbol interference from the earlier symbol. Therefore 16 bits are added at the starting of each IFFT output to get total of 80 bits per symbol.

Basically, a filter can be used to reject all unwanted signal components and pass only those signals which are to be allowed for the further processing. The output of the transmitter chain signal is a weak signal, for base band wireless applications strong signal strength should be neccesary because it will travel for long distances during wireless transmissions. So, PA strengthens the weak signal from $67 \mathrm{dBm}$ to $122 \mathrm{dBm}$ with $50 \%$ efficiency and $55 \mathrm{~dB}$ gain. The PS splits output of the PA $122 \mathrm{dBm}$ into two equal signals of $122 \mathrm{dBm}$ each. By using PML concept [15] the loss less power dividing is possible that is input $122 \mathrm{dBm}$ is purely obtained at the output. The Rayleigh fading attenuates nearly $67 \mathrm{dBm}$ power from $122 \mathrm{dBm}$ to get $54 \mathrm{dBm}$ power because of the multipath distortion. AWGN is simply adds uniformly distributed white Gaussian noise to the output of Rayleigh fading which gives the signal to noise ratio $12 \mathrm{~dB}$.

The signal strength of LNA is increased from $54 \mathrm{dBm}$ to $67 \mathrm{dBm}$ which is nearly equal to the output signal of the transmitter chain and the SNR is increased to $21 \mathrm{~dB}$ which much better than the wireless channel. The maximum available gain of LNA is $53 \mathrm{~dB}$. ARC output directly taken from PS which is a wired communication, but ORC output is taken from wireless channel. Due to attenuation losses in wireless channel ORC amplitude value is very small when compared to ARC. It has very low quantization noise nearly equal to zero because of high number of bits (80000). The time domain samples are converted into frequency domain samples for analysis of complete system. LS channel estimation is very easy technique to implement but it has no perfect channel information. So, proposed modified DFT based channel estimation is used to improve channel information. The MSE value for LS channel estimation technique is very large because it does not have any channel information. So, MSE is decreased to a satisfactory level by using the DFT based channel estimation technique which divides the maximum length of channel taps by output of LS channel estimator. Now, by using proposed modified DFT 
based channel estimation technique MSE value is decreased by a large extent as displayed in the below fig 4 .

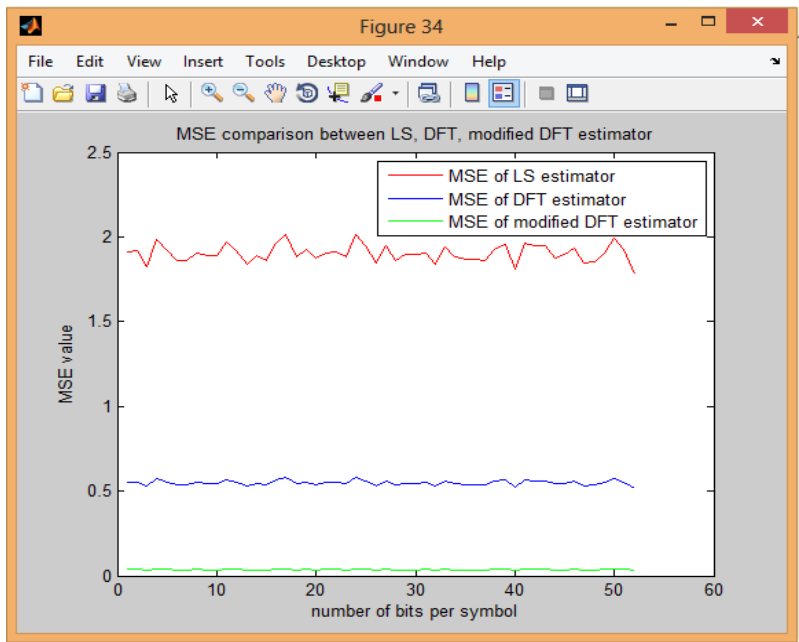

Fig 4: MSE comparison between LS, DFT and Modified DFT channel estimators

The noise floor has $-90 \mathrm{dBm}$ self-interference signal which is taken as reference signal. By using the digital cancellation technique the SISO self-interference cancellation signal is -87 $\mathrm{dBm}$ which is a $3 \mathrm{dBm}$ above the noise floor. Now, the obtained proposed MIMO self-interference cancellation signal is $-53 \mathrm{dBm}$ which is a $57 \mathrm{dBm}$ above the noise floor. The proposed technique is $54 \mathrm{dBm}$ above the previous SISO full duplex system technique.

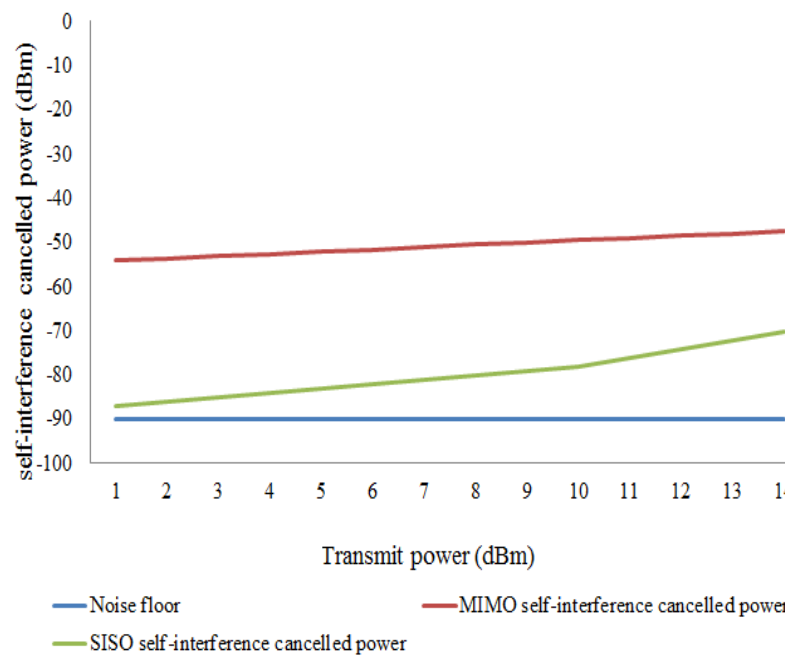

Fig 5: self-interfernce cancellation comparison between noise floor, MIMO system and SISO system

The SNR of the channel is very low that is $8 \mathrm{~dB}$ when compared to the LNA and channel estimation due to attenution and phase losses by the rayleigh fading. So, by using the matched network circuits and S-parameter model the reduced signal is increased to required value and its signal to noise ratio is increased to $14 \mathrm{~dB}$. By using proposed modified DFT based channel estimation technique the SNR is increased to $20 \mathrm{~dB}$ which is high value when compared to channel and LNA outputs.

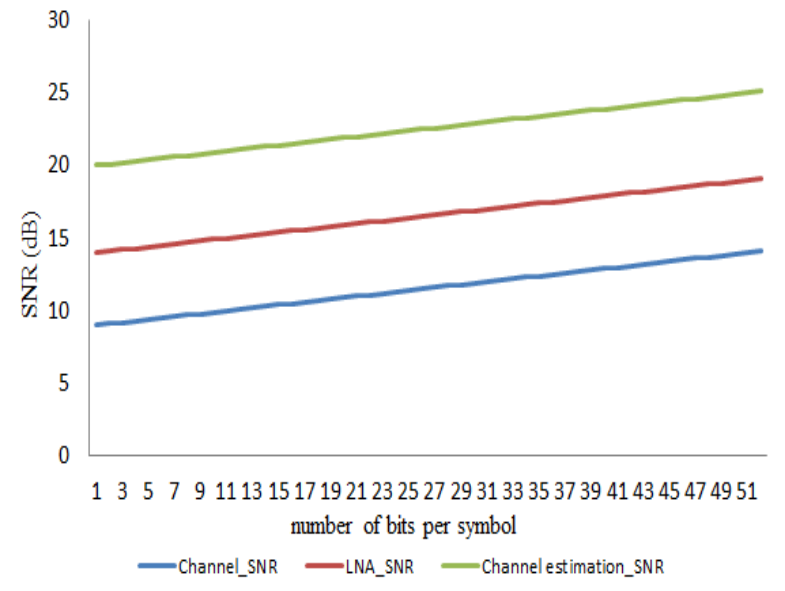

Fig 6: SNR comparison between channel, LNA and channel estimation

\section{CALCULATIONS of SYSTEM PARAMETERS}

\subsection{Calculation of Achievable Rate}

The obtained achievable rate for the MIMO full duplex system is $28 \mathrm{bits} / \mathrm{sec} / \mathrm{Hz}$ which is higher than MIMO halfduplex system $14 \mathrm{bits} / \mathrm{sec} / \mathrm{Hz}$, SISO full duplex system 10 bit/sec/Hz and SISO full duplex system $5 \mathrm{bit} / \mathrm{sec} / \mathrm{Hz}$ as displayed in the below fig 7 .

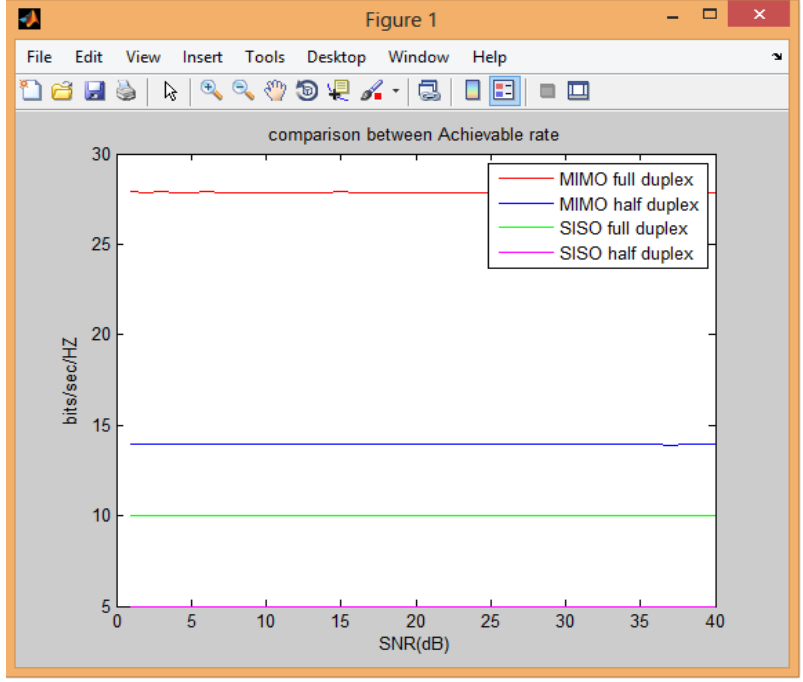

Fig 7: Achievable rate comparison between MIMO full duplex, MIMO half duplex, SISO full duplex and SISO half duplex systems

\subsection{Calculation of Channel Capacity}

The obtained channel capacity for the MIMO full duplex system is $560000000 \mathrm{bits} / \mathrm{sec}$ (534.05 Mbps) which is higher speed than MIMO half-duplex system $280000000 \mathrm{bits} / \mathrm{sec}$ (267.02 Mbps ), SISO full duplex system $200000000 \mathrm{bit} / \mathrm{sec}$ (190.73 Mbps) and SISO half-duplex system 100000000 bit/sec (95.36 Mbps) as displayed in the below fig 8 . 


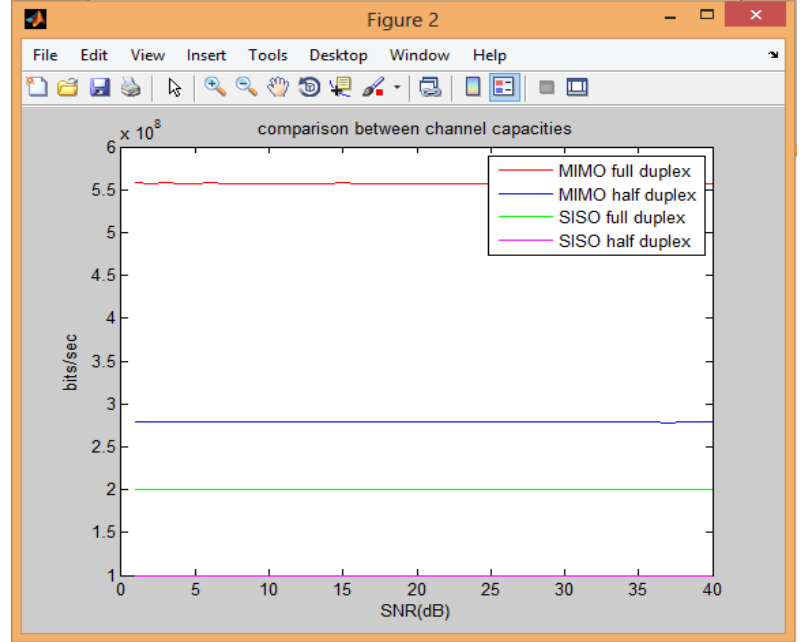

Fig 8: Channel capacity comparison between MIMO full duplex, MIMO half duplex, SISO full duplex and SISO half duplex systems

\subsection{Output of Throughput}

The obtained throughput for the MIMO full duplex system is $170000000 \mathrm{bits} / \mathrm{sec}(162.12 \mathrm{Mbps})$ which is higher speed than MIMO half-duplex system 120000000 bits/sec (81.06 Mbps ), SISO full duplex system $20000000 \mathrm{bit} / \mathrm{sec}(19.07 \mathrm{Mbps})$ and SISO half-duplex system $10000000 \mathrm{bit} / \mathrm{sec}$ (9.53 Mbps)as displayed in below fig 9 .

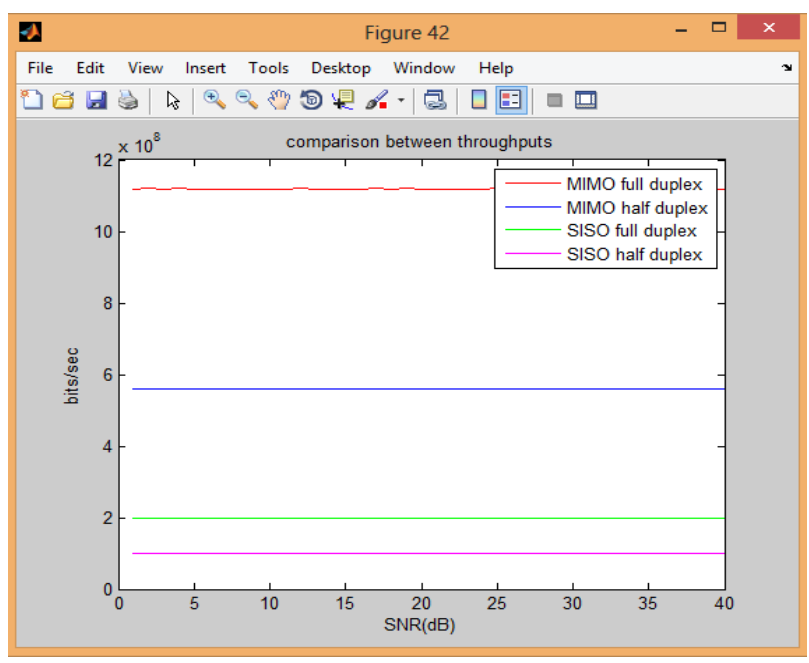

Fig 9: Throughput comparison between MIMO full duplex, MIMO half duplex, SISO full duplex and SISO half duplex systems

\subsection{Output of Spectral Efficiency}

The obtained spectral efficiency for the MIMO full duplex system is $82 \%$ which is higher efficiency than SISO full duplex system $76 \%$ as displayed in the below fig 10 .

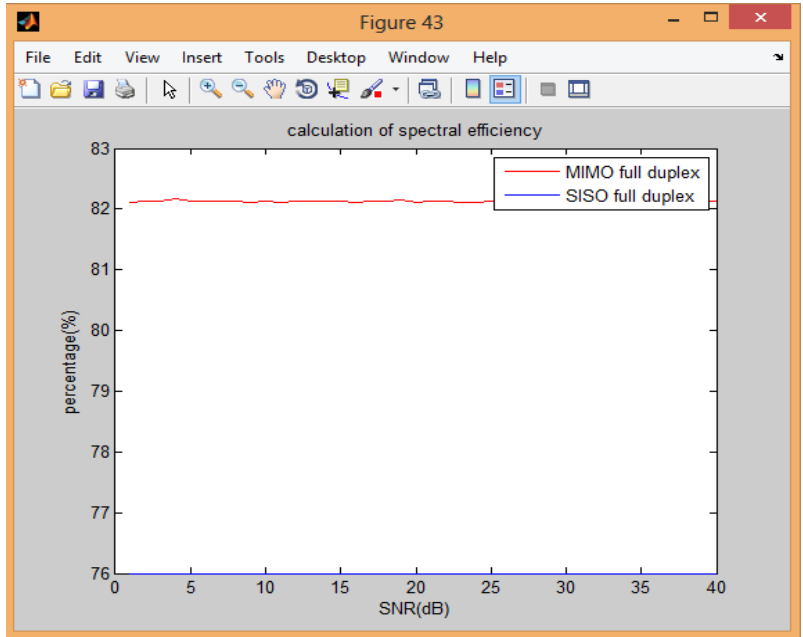

Fig 10: spectral efficiency comparison between MIMO full duplex, MIMO half duplex, SISO full duplex and SISO half duplex systems

The channel capacity and throughput of MIMO full duplex system is large when compared to MIMO half duplex , SISO full duplex and SISO half duplex systems. So, MIMO full duplex system delivers high data rate when compared to other systems as displayed in the below table 1 .

Table 1. Achievable rate, channel capacity and throughput comparison between MIMO full duplex, MIMO half duplex, SISO full duplex and SISO half duplex systems

\begin{tabular}{|c|c|c|c|c|}
\hline Type of system & SNR (dB) & $\begin{array}{c}\text { Achievable rate } \\
\text { (bits/sec/Hz) }\end{array}$ & $\begin{array}{c}\text { Channel } \\
\text { capacity (Mbps) }\end{array}$ & $\begin{array}{c}\text { Throughput } \\
\text { (Mbps) }\end{array}$ \\
\hline $\begin{array}{c}\text { MIM0 full duplex } \\
\text { system } \\
\text { (proposed method) }\end{array}$ & 40 & 28 & 534.05 & 1049.04 \\
\hline $\begin{array}{c}\text { MIM0 half-duplex } \\
\text { system }\end{array}$ & 40 & 14 & 267.02 & 524.50 \\
\hline $\begin{array}{c}\text { SISO full duplex } \\
\text { system }\end{array}$ & 40 & 10 & 190.73 & 190.73 \\
\hline $\begin{array}{c}\text { SISO half-duplex } \\
\text { system }\end{array}$ & 40 & 5 & 95.36 & 95.36 \\
\hline
\end{tabular}

\section{CONCLUSION}

The designed MIMO full-duplex system uses QAM for information carried out in both amplitude and phase of the transmitter carrier. By using OFDM technique the symbols created by the QAM travels orthogonally along the wireless channel and digital domain signal is converted into analog domain signal for wireless base band applications. PA in the transmitter side strengthens the signal to a large extent and splits the strengthened signal equally into wired and wireless channels. When the signal passes through wireless channel its strength decreases and noise added due to the Rayleigh fading and AWGN.

LNA in the receiver section increases the signal strength to a required value and converts into the digital signal for digital applications. The receiver section consists of auxiliary chain and its copy circuit called ordinary chain is given to channel estimation which reduces the noise to an optimized value. By using proposed modified DFT based channel estimation technique the amount of self-interference cancelled is $57 \mathrm{dBm}$ higher than the receiver floor. The achievable rate and 
throughput of MIMO full duplex system is $28 \mathrm{bits} / \mathrm{sec} / \mathrm{Hz}$ and 1049.04 Mbps respectively which is very large when compared to MIMO half duplex, SISO full duplex and SISO half duplex systems.

\section{REFERENCES}

[1] E. Ahmed and A. M. Eltawil, "All-Digital SelfInterference Cancellation Technique for Full-Duplex Systems," IEEE Trans. Wireless Commun., VOL. 14, NO. 7, pp. 3519-3532, Jul. 2015.

[2] Zhongshan Zhang, Xiaomeng Chai, Keping Long, Athanasios V. Vasilakos, and Lajos Hanzo, "Full Duplex Techniques for 5G Networks: Self-Interference Cancellation, Protocol Design, and Relay Selection," IEEE Communications Magazine, pp. 2-10, May 2015.

[3] E. Ahmed and A. M. Eltawil, "On Phase Noise Suppression in Full-Duplex Systems," IEEE Trans. Wireless Commun., VOL. 14, NO. 3, pp. 1237-1251, Mar. 2015

[4] E. Everett, A. Sahai, and A. Sabharwal, "Passive Selfinterference Suppression for Full-Duplex Infrastructure Nodes," IEEE Trans. Wireless Commun., VOL. 13, NO. 2, pp. 680-694, Feb. 2014.

[5] M. Duarte et al., "Design and Characterization of a FullDuplex Multiantenna System for WiFi Networks," IEEE Trans. Veh. Technol., VOL. 63, NO. 3, pp. 1160-1177, Mar. 2014.

[6] Juhi Garg, Kapil Gupta, P. K. Ghosh, "Performance Analysis of MIMO Wireless Communications over Fading Channels - A Review," International Journal of Advanced Research in Electrical, Electronics and Instrumentation Engineering, VOL. 2, NO. 4, pp. 12721302, Apr. 2014.

[7] A. Sahai, G. Patel, C. Dick, and A. Sabharwal, "On the Impact of Phase Noise on Active Cancelation in Wireless Full-Duplex," IEEE Trans. on vehicular technology, VOL. 62, NO. 9, pp. 4494-4510, Nov. 2013.
[8] D. Abhijit, V. Prasanth, "OFDM System Using FFT and IFFT ," International Journal of Advanced Research in Computer Science and Software Engineering, VOL. 13, NO. 12, pp. 675-679, Dec. 2013.

[9] Nuzhat Tasneem Awon, Md. Mizanur Rahman, "Effect of AWGN \& Fading (Raleigh \& Rician) Channels on BER Performance of a WiMAX Communication System," International Journal of Computer Science and Information Security, VOL. 10, NO. 8, pp. 11-17, Aug. 2012.

[10] Ifeagwu E.N., Ekehj, Ohaneme C.O., Okezie C.C., "Evaluation of Spectral Efficiency, System Capacity and Interference Effects on CDMA Communication System," International Journal of Advanced Computer Science and Applications, VOL. 3, NO. 6, pp. 20-25, Dec. 2012.

[11] M.Muhamad, N.A.Nordin, "S-Parameter Analysis of $0.18 \mu \mathrm{m}$ LNA for WCDMA Application," International Conference on Microelectronics, Nanoelectronics, Optoelectronics, pp.48-51, Aug. 2010.

[12] Aripirala Manoj Kumar, G. Bharath Reddy, G. Krishna Chaitanya Reddy, "Modelling RF Power Amplifier to Study its Nonlinear Effects on RF Communication System, with BER as a Performance Measure," International Journal of Next-Generation Networks, vol. 2, no. 4, pp. 73-81, Dec. 2010.

[13] A. Ghaffari, F. monifi, M. djavid, "Analysis of Photonic Crystal Power Splitters with Different Configurations," journal of applied sciences, VOL. 8, NO. 8, pp. 14161425, Aug. 2008

[14] Y. Kang, K. Kim and H. Park, "Efficient DFT-based Channel Estimation for OFDM Systems on Multipath Channels," IET Commun., vol. 1, no. 2, pp. 197-202, Apr. 2008.

[15] Jean-Pierre Berenger, "A Perfectly Matched Layer for the Absorption of Electromagnetic Waves," journal of computational physics, pp. 185-200, Jul. 1994. 